\title{
Research on the ecological restoration method for highway debris slope: A case study of Guidu Highway in China
}

\author{
Shu Liu ${ }^{1}$, Nan Zhang ${ }^{2}$, Xiaochun Qin ${ }^{2}$, Yaokai Wang ${ }^{2}$ \\ ${ }^{1}$ Appraisal Center for Environment \& Engineering, Ministry of Environmental Restoration, Beijing, 100012, China \\ ${ }^{2}$ School of civil and architectural engineering, Beijing Jiaotong University, Beijing, 100044, China
}

\begin{abstract}
To clarify the superiority of slope ecological restoration technology in slope restoration, the artificial slope in the section of Guiyang-Duyun highway as the case study was analyzed in this paper. Plant sample collection experiments were carried out by sample method, and the evaluation indicators factors were plant distribution density, biomass and soil nutrients. The effect of slope restoration was evaluated by comparing with the ecological effect of traditional slope restoration measures. The results showed that for the slope ecological restoration sample, the average cumulative plant density was 187 plants per square meter, about 3.8 times of the comparison sample. The average biomass was $333.25 \mathrm{~g} / \mathrm{m}^{2}$, which was about 6.4 times that of the comparison sample. Soil nutrients were also much higher than those in the comparison sample. The ecological restoration of the slope with ProGanics and Flexterra materials was superior to the traditional slope restoration measures. The oretical research results will have important theoretical and technical significance for the ecological restoration method for highway debris slope of Guidu highway.
\end{abstract}

\section{Introduction}

The history of slope ecological restoration technology was very long in European and American countries, developed countries such as the United States had long realized the importance of slope ecological restoration measures to protect the ecology of highway areas. In 1943, the American professor Moorish R.H ${ }^{[1]}$ took the experiment of planting sod on two sides of highway and took the research on planting time, grass species and grass seed combination, discussed the way of sod revetment. In 1986, the Mcelroy M.T. ${ }^{[2]}$ who was in American conducted systematic research on management and conservation of vegetation slopes. In 1973, Japan developed the Fiber-soil Greening Method. R. Bhattacharyya, M.A. Fullen ${ }^{[3]}$ and other scholars studied the application of palm-pad geotechnical cloth as a rainwater erosion control technology. Pablo García-Palacios $^{[4]}$ and other scholars worked out the conclusion that the best restoration when mixing herb and shrub plant species for slope ecological restoration. M. Fattet ${ }^{[5]}$ and other scholars found that the plant mixing of different functional types could improve soil restoration by reducing surface water erosion and light matrix mass movement. Ruth C. Martín-Sanz ${ }^{[6]}$ and other scholars showed that in a relatively short period of time, adjacent seed sources existed in the residual plaques of natural vegetation, and the vegetation communities that grew in natural conditions were more abundant. Alexandra Medi[ ${ }^{[7]}$ and other scholars proved that the use of geotechnical grids and coconut shell nets were of the best use and were very useful in the greening of high and steep slopes.

The time when we started to research which was aimed at the slope ecological restoration technology in China was a little bit later. In 1994, Li Xuguang ${ }^{[8]}$ explored the construction technical points of SF ecological restoration method and mud spray ecological restoration method. In 2000, Zhang Mengtao ${ }^{[9]}$ and others carried out a plant concrete study. In 2001, Jin Zhong ${ }^{[10]}$ obtained that the spray planting technology had good ecological benefits. In 2009, Sun Chao and $\mathrm{Xu}$ Wennian $^{[11]}$ and others introduced PEB ecological slope restoration technology in the highway slope ecological restoration project. Li Leibo and Chen Mingxu ${ }^{[12]}$ and other scholars found the optimal amount of high absorbent resin. Yang Yang and Yang Jianying ${ }^{[13]}$ and other scholars investigated the effects of soil moisture, soil temperature and vegetation growth on soil physical and chemical properties through laboratory experiments. Yan Ping and other scholars ${ }^{[14]}$ explored the new ecological restoration technology for different rock matter and slope rate of the applicability. Liu Xinxin ${ }^{[15]}$ analyzed the multi-faceted impact of highway engineering construction on the ecological environment. 


\section{Methods and materials}

\subsection{Study on selection of resistant plants and ratio of seed dosage.}

2.1.1 Study on the resistance of plants. In order to resist the stress of the living environment, in the long-term natural selection, the good characteristics of plants were preserved, but these were often specific, and cannot fully meet people's needs, therefore, people need to improve the resistance of plants.

2.1.2 Selection of resistant plants. As far as the slope of this experiment was concerned, the soil quality was mostly poor, and so we should choose drought-resistant, barren-resistant, deep root-based grass irrigation plants. Therefore, this project used hedgehogs, multi-flower magnolias, multi-flowered husks, double-twisted, fire-thorns, yellow flowers, pig poo beans, dog root, white three-leaf, rye grass, sliver shear, tie grass and wildflower combination.

2.1.3 Study on the dosage and proportion of plant seeds. The seeding calculation formula used in this experiment was:

$$
W=\frac{A \times F}{1000 \times C \times E \times P}
$$

In: $\mathrm{W}$ was the seeding amount $\left(\mathrm{g} / \mathrm{m}^{2}\right)$ of plant species; A was the expected number of plants (strains $/ \mathrm{m}^{2}$ ); C was the correction rate of standing conditions; E was the seed germination rate; F was the seed's kilogram weight (g); P was the purity of the seed.

Table 1.The allocation and seeding rate of plant

\begin{tabular}{cc}
\hline Name & Dosage $\left(\mathrm{g} / \mathrm{m}^{2}\right)$ \\
\hline Robinia pseudoacacia & 6 \\
Magnolia multiflora & 5 \\
Lespedeza floribunda Bunge & 4 \\
Cassia bicapsularis & 3 \\
Pyracantha fortuneana & 4 \\
Sophora xanthantha & 4 \\
Crotalaria mucronata & 5 \\
Bermuda grass & 3 \\
Trifolium repens & 2 \\
Ryegrass & 2 \\
\hline
\end{tabular}
Agrostis stolonifera
1.5
Zoysia japonica
Wildflowers
2

\subsection{Study on the proportion of topsoil material and the application of anti-erosion fiber material}

2.2.1 Components and functions of topsoil materials. In the actual project, the topsoil material was mainly composed of the following parts:

(1) Planting soil: it used to make the three-phase distribution of the substrate mixture more reasonable.

(2) Fiber material: it used to help improve the interaction between the components of the substrate mixture.

(3) Organic matter: the main role of organic matter was to make a reasonable substrate mixture structure more easily formed.

(4) Spray water: spray water should choose non-polluting water sources.

(5) Stabilizer, non-polluting binder: the polymer and natural plant processing were used.

(6) Fertilizer: fertilizer mainly used compound fertilize.

\subsubsection{Topsoil fibrous material-ProGanics. It fundamentally improved the proportion of soil composition, and then improved the soil structure, improved the soil's own ability to maintain water and fertilizer. TM contained the main ingredients as follows: recyclable heat-treated wood fibers, biochar, polysaccharides, seaweed essence, humic acid and other additives.}

\subsubsection{Anti-erosion fiber material -Flexterra.} Protecting slope soil will not cause soil erosion due to rain or other runoff, was a completely biodegradable and effective anti-rainwater-resistant ecological material. The ingredients of Flexterra were as follows: heat-treated log fibers, moisturizing ingredients, curly biodegradable artificial interlocking fibers, microporous particles.

\subsubsection{Topsoil material and anti-erosion fiber material ratio.}

Table 2.The proportion of topsoil material and anti - erosion fiber material

\begin{tabular}{ccc}
\hline Name & Dosage & Notes \\
\hline Plant generates & $0.05 \mathrm{~m}^{3} / \mathrm{m} 2$ & Add organic fertilizer, compound fertilizer, water retention agent, adhesive \\
ProGanics & $600 \mathrm{~g} / \mathrm{m} 2$ & Field-of-sight conditions, appropriate adjustments \\
Flexterra & $400 \mathrm{~g} / \mathrm{m} 2$ & Field-of-sight conditions, appropriate adjustments \\
Compound fertilizer & $30 \mathrm{~g} / \mathrm{m} 2$ & Akon \\
Organic material & $150 \mathrm{~g} / \mathrm{m} 2$ & Organic matter accounts for 3\%, animal or peat soil \\
\hline
\end{tabular}




\section{A comparative study on the application effect of the ecological restoration technology on the slope}

\subsection{On-site and indoor study studies}

3.1.1 Plant sample survey study. In the course of this experiment, a plant sample collection experiment was carried out using $1 \mathrm{~m} \times 1 \mathrm{~m}$ square sample, and three sample squares were selected in the study area for the collection of plant biomass and soil nutrient determination samples and the determination of plant distribution density. Samples and experiments were collected and carried out in the same period in areas

3.1.3 Table 3 about the main experimental equipment used and the main indicators of the measurement and It is shown in Table 4 about the determination. Plant biomass determination experiments were mainly based on the fresh weight and dry weight value of plant samples collected at the site using drying equipment and high-precision electronic balance determination site, and it is shown in Table 5 about the main instruments used: where the traditional method of slope vegetation restoration was carried out at a distance of about $1000 \mathrm{~m}$.

To prevent omissions, $20 \mathrm{~cm}$ were spaced on the identified samples and counted in order from the top left to right, from the top to bottom.

3.1.2 Indoor study. Indoor experiments mainly included soil nutrient determination study and plant biomass determination study. The soil nutrient determination experiment was mainly used in the laboratory to determine the various nutrient indexes obtained from the field by the field. It is shown in

Table 3.The index and instrument of soil nutrient determination experiment

\begin{tabular}{ccc}
\hline Sample name & Soil \\
\hline Sample status & Solid particles & Sample size \\
Study index & $\begin{array}{r}\text { PH, Moisture, Total nitrogen, Total phosphorus, Total potassium, Hydrolysis of sex } \\
\text { of nitrogen, Rapidly available potassium, Organic matter, Available phosphorus }\end{array}$ \\
Main instrument & EN-130 FE28 Portable acidity meter, EN-141 ME2002 Electronic scales \\
EN-142 ML204T Electronic scales, EN-134 ME204TE Electronic scales \\
EN-137 AA Series Flame atom absorbs spectrophotometer, \\
EN-139 V1000 Visible light spectrophotometer, \\
EN-117 DHG-9245A Electric heat-constant drum wind drying box, \\
EN-128 LRH-250 Biochemical incubator
\end{tabular}

Table 4.The study basic of soil nutrient determination experiment

\begin{tabular}{|c|c|c|}
\hline \multirow{2}{*}{ Study project } & \multicolumn{2}{|r|}{ Study standard } \\
\hline & Study standard & Standard method \\
\hline $\mathrm{PH}$ & NY/T 1121.2-2006 & Soil Studying-Part II: Determination of Soil PH \\
\hline Total nitrogen & HJ 717-2014 & $\begin{array}{l}\text { Soil quality-Determination of whole } \\
\text { nitrogen-Kjeldahl }\end{array}$ \\
\hline Total potassium & NY/T87-1988 & Soil-Whole potassium assay \\
\hline Total phosphorus & NY/T88-1988 & Soil-Whole phosphorus assay \\
\hline Moisture & HJ 613-2011 & $\begin{array}{l}\text { Soil-Determination of dry matter and } \\
\text { moisture-Gravimetric method }\end{array}$ \\
\hline $\begin{array}{c}\text { Hydrolysis of sex of } \\
\text { nitrogen }\end{array}$ & $\mathrm{LY} / \mathrm{T} 1228-2015$ & Determination of nitrogen in forest soil \\
\hline
\end{tabular}


Organic matter

Available phosphorus

Rapidly available potassium
NY/T 1121.6-2006

$\mathrm{NY} / \mathrm{T} 1121.7-2014$

NY/T 889-2004
Soil detection-Part 6: Determination of soil organic matter

Soil detection-Part 6: Determination of soil organophosphate

Determination of soil quick-acting potassium and slow-acting potassium content

Table 5.The experimental apparatus for determination of plant biomass

\begin{tabular}{|c|c|c|c|}
\hline Sample name & \multicolumn{3}{|c|}{ Botany } \\
\hline Sample status & Fresh plant sample & Simple size & $12 \mathrm{bag}$ \\
\hline Study project & \multicolumn{3}{|c|}{ Vegetation biomass } \\
\hline Main instrument & $\begin{array}{r}\text { EN-142 ML2 } \\
\text { EN-117 D }\end{array}$ & $\begin{array}{l}\text { ectronic scales } \\
5 \mathrm{~A} \text { Electric h }\end{array}$ & $\begin{array}{l}\text { lectronic scales } \\
\text { d drying box }\end{array}$ \\
\hline
\end{tabular}

\subsection{A comparative study of plant growth effect indicators}

\subsubsection{Comparison of plant distribution density}

Table 6.Statistical table of slope plant density

\begin{tabular}{cccccc}
\hline \multirow{2}{*}{$\begin{array}{c}\text { Serial } \\
\text { number }\end{array}$} & & \multicolumn{4}{c}{ density $\left(\right.$ Plant $\left./ \mathrm{m}^{2}\right)$} \\
\cline { 3 - 6 } & & Quadrat one & Quadrat two & Quadrat there & $\begin{array}{c}\text { Compare the } \\
\text { quadrat }\end{array}$ \\
\hline 1 & Robiniapseudoacacia & 0 & 0 & 0 & 0 \\
2 & Magnolia multiflora & 3 & 4 & 3 & 0 \\
3 & Lespedeza floribunda Bunge & 5 & 7 & 8 & 0 \\
4 & Cassia bicapsularis & 2 & 1 & 2 & 0 \\
5 & Pyracanthafortuneana & 0 & 0 & 0 & 0 \\
6 & Sophoraxanthantha & 1 & 0 & 2 & 0 \\
7 & Crotalaria mucronata & 45 & 39 & 42 & 0 \\
8 & Bermuda grass & 19 & 24 & 21 & 0 \\
9 & Trifoliumrepens & 26 & 24 & 30 & 6 \\
10 & Ryegrass & 49 & 43 & 52 & 12 \\
11 & Agrostisstolonifera & 6 & 5 & 2 & 11 \\
12 & Zoysia japonica & 31 & 29 & 35 & 19 \\
13 & Wildflowers & 0 & 0 & 0 & 0 \\
& Total & 187 & 176 & 197 & 48 \\
\hline
\end{tabular}

It is shown in Table 6 that the cumulative plant density at the sample siphons was 187 strains $/ \mathrm{m}^{2}$, respectively, 176 strains $/ \mathrm{m}^{2}$ and 197 plants $/ \mathrm{m}^{2}$, significantly higher than the contrasting sample of 48 plants $/ \mathrm{m}^{2}$, indicating that $\mathrm{TM}$ and Flexterra materials were more conducive to plant growth, in the early stage can better ensure the survival rate of plants, thereby ensuring the growth density of plants later in the period.
3.2.2 Plant Biomass Comparison Analysis. In this experiment, only the biomass of the plant ground part was measured to compare the growth of plants at different locations.

The value of plant biomass at the sample siphons of one, two and three were significantly higher than the value of biomass at the contrasting sample. It is shown that when the slope ecological protection is used with ProGanics and Futra materials, it is more helpful to the growth of plants, which can help the ecological 
protection of slope.

Table 7.Statistical table of sample plot biomass measurement results

\begin{tabular}{c|c|c|c}
\hline Quadrat one & Quadrat two & Quadrat there & Compare the quadrat \\
\hline 334.22 & 338.7 & 326.84 & 51.86 \\
\hline
\end{tabular}

\subsubsection{Comparison of nutrients in slope soil. Soil} nutrients were essential nutrients for plant growth provided by the soil. It is shown in

3.2.4 Table 8 that the study project and the study results value of the soil sample in this experiment.

Table 8.Statistical table of soil nutrient determination results

\begin{tabular}{|c|c|c|c|c|c|}
\hline Study project & Quadrat one & Quadrat two & Quadrat there & $\begin{array}{c}\text { Compare the } \\
\text { quadrat }\end{array}$ & Unit \\
\hline $\mathrm{PH}$ & 7.79 & 7.27 & 7.38 & 6.68 & dimensionless \\
\hline Total nitrogen & 2010 & 1930 & 1951 & 824 & $\mathrm{mg} / \mathrm{kg}$ \\
\hline Total potassium & 1.47 & 1.16 & 1.35 & 0.64 & $\%$ \\
\hline Total phosphorus & 0.061 & 0.062 & 0.046 & 0.031 & $\%$ \\
\hline Moisture & 18 & 14 & 15 & 8 & $\%$ \\
\hline $\begin{array}{l}\text { Hydrolysis of sex of } \\
\text { nitrogen }\end{array}$ & 100 & 122 & 125.9 & 49 & $\mathrm{mg} / \mathrm{kg}$ \\
\hline Organic matter & 74000 & 75500 & 69600 & 21800 & $\mathrm{mg} / \mathrm{kg}$ \\
\hline Available phosphorus & 45.2 & 36.8 & 39.6 & 14.2 & $\mathrm{mg} / \mathrm{kg}$ \\
\hline $\begin{array}{l}\text { Rapidly available } \\
\text { potassium }\end{array}$ & 256 & 311 & 283 & 85 & $\mathrm{mg} / \mathrm{kg}$ \\
\hline
\end{tabular}

By analyzing this chart, it can be seen that the content of the slope ecological restoration technology carried out by ProGanics and Flexterra materials were significantly higher than that of the whole nitrogen, hydrolytic nitrogen, organic matter, effective phosphorus and quick-acting potassium after a certain period of time, among which the difference in the content of organic matter was more obvious. Up to three times the construction pattern of ordinary slope restoration technology indicates that ProGanics and Flexterra materials were rich in organic matter, thus providing a more adequate supply of nutrients for plant growth on the slope; and the use of slope ecological restoration technology of soil moisture content was significantly higher than the traditional slope restoration technology sample, indicating that ProGanics and Flexterra materials helped soil moisture conservation, and contributed to the growth of plants.

\section{Conclusion}

(1) The anti-reversal and dosage ratio studies of plants were carried out through the principle of anti-reversal plant selection, and the type and dosage of plant seeds were suitable for the experimental slope of the plant was determined, and the experimental results showed that the selected plant had excellent resistance and reasonable ratio, which contributed to the establishment of the slope plant community;

(2) By comparison, the study found that the ecological effect observation index of the protective slope was obviously different. The superiority of plant biomass in slope ecological restoration technology was the highest, and the distribution density was obvious. Obviously, when using ProGanics and Flexterra materials for slope ecological restoration, it was superior to the traditional slope restoration technology ecological benefits, indicating that the topsoil materials ProGanics and anti-erosion fiber material Flexterra had achieved the expected experimental effect, which was very practical for the ecological restoration of highways in operation.

(3) Theoretical research results will have important theoretical and technical significance for the ecological restoration method for highway debris slope of Guidu highway.

\section{References}

1. Moorish RH. The establishment and comparative we are resistance of various grasses and grass legume mixture to vehicular traffic. Highway Res $\mathrm{Bd}$ Highwayside, Dev.Com. Report,1989.

2. McElroy MT, Pieke PE, McBurney SL. Utilizing plant growth regulators to develop acost efficient 
management system for highwayside vegetation, 1984.

3. Bhattacharyya R, Fullen M A, Davies K, et al. Use of palm-mat geotextiles for rainsplash erosion control.[J]. Geomorphology, 2010, 119(1-2):52-61.

4. García-Palacios P, Soliveres S, Maestre F T, et al. Dominant plant species modulate responses to hydroseeding, irrigation and fertilization during the restoration of semiarid motorway slopes[J]. Ecological Engineering, 2010, 36(10):1290-1298.

5. Fattet $\mathrm{M}, \mathrm{Fu} \mathrm{Y}$, Ghestem $\mathrm{M}$, et al. Effects of vegetation type on soil resistance to erosion: Relationship between aggregate stability and shear strength[J]. Catena, 2011, 87(1):60-69.

6. Martín-Sanz R C, Fernández-Santos B, Martínez-Ruiz C. Early dynamics of natural revegetation on highwaycuts of the Salamanca province (CW Spain)[J]. Ecological Engineering, 2015, 75(75):223-231.

7. Medl A, Stangl R, Kikuta S B, et al. Vegetation establishment on 'Green Walls': Integrating shotcrete walls from highway construction into the landscape[J]. Urban Forestry \& Urban Greening, 2017, 25.

8. Li Xuguang, Mao Wenbi, XuFuyou. Greening and Restoration of Highway Slopes in Japan-- A Study Report on A Visit to Japan in 1994 [J]. Highway Traffic Technology, 1995, 12 (2): 59-64.

9. Shu Xiang, Cao Yinghui. Building a Green Channel And Taking the Highway to Environmental Restoration -- The Practice of Comprehensive Management of the Slope of the HuiheHighway[J]. Highway, 2001 (2):79-83.

10. Jin Zhong. Experimental application of spray-planting technology in the restoration of high slope of HuiheHighway[J].Guangdong Highway Traffic, 2001 (2):18-19.

11. Sun Chao, XuWennian, Zhou Mingtao, et al. Research and application of anti-flushing substrate ecological slope restoration technology [J]. Water and Hydropower Technology, 2009, 40 (1):37-40.

12. Li L, Chen M, Zhou X, et al. A case of water absorption and water/fertilizer retention performance of super absorbent polymer modified sulphoaluminatecementitious materials[J]. Construction \& Building Materials, 2017, 150:538-546.

13. Yang Y, Yang J, Zhao T, et al. Ecological restoration of highway slope by covering with straw-mat and seeding with grass-legume mixture[J]. Ecological Engineering, 2016, 90:68-76.

14. Yan Ping, Wang Yanwing, Yi Weifeng, et al. New ecological restoration technology to deal with soft rock slope applicability research [J].Sino-foreign highway, 2018 (6): 29-33.

15. Liu Xinxin. Study on the restoration technology of ecological highway slope [J]. Transport World, 2019 (07): 26-27. 